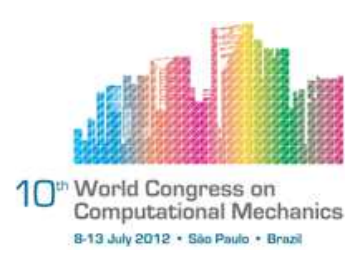

\title{
A STABILIZED HYBRID FINITE ELEMENT METHOD FOR THE LINEAR ELASTICITY PROBLEMS
}

\author{
C. O. Faria ${ }^{1}$, A. J. Boness ${ }^{2}$ and A. F. D. Loula ${ }^{1}$ \\ ${ }^{1}$ Laboratório Nacional de Computação Científica, LNCC/MCTI (cofaria@ lncc.br) \\ ${ }^{2}$ Universidade Federal Rural do Rio de Janeiro, UFRRJ
}

Hybrid formulations have been widely used in computational mechanics associated with primal or mixed finite element methods. Recently, hybrid formulations have been developed associated with Discontinuous Galerkin methods. In this work we propose a new primal hybrid finite element method for linear elasticity. Using a stabilization strategy typical of Discontinuous Galerkin methods, we choose as multiplier the displacement field itself and add stabilization and symmetrization terms to generate a stable and adjoint consistent formulation allowing greater flexibility in the choice of basis functions of approximation spaces for the displacement field and the Lagrange multiplier. The local problems, in the displacement field, can always be solved at the element in favor of the Lagrange multiplier defined on each edge of the elements. The global system is assembled involving only the degrees of freedom associated with the Lagrange multipliers, as usual in a hybrid method, where the continuity on the element edges is imposed weakly. Polynomial bases are adopted to approximate both the displacement field and the Lagrange multipliers considering Lagrangian polynomial base. Result of some numerical experiments are presented to illustrate the potential of the proposed formulation.

Keywords: Linear Elasticity, Discontinuous Galerkin, Hybridization, Stabilization.

\section{INTRODUCTION}

It is well known that standard Galerkin finite element approximations for elasticity problem degrade when the Poisson's ratio tends to $1 / 2$, corresponding to near incompressible elasticity. This nonrobustness of the FEM is widely termed "locking". To avoid the locking effects, several approaches have been introduced. We mention here the mixed finite element methods in $[4,10,11]$, the nonconforming methods proposed in [14], the higher-order methods in [18] and the Discontinuous Galekin (DG) methods in [17, 13, 19, 5]. All these methods have been quite extensively studied within an a priori context.

Robustness and flexibility for implementing $h$ and $p$-adaptivity strategies are well known advantages of Discontinuous Galerkin (DG) methods stemming from the use of finite element spaces consisting of discontinuous piecewise polynomials. The natural connection between DG formulations and hybrid methods have been successfully exploited to derive new finite element methods with improved stability and reduced computational cost but still keeping the robustness and flexibility of DG methods $[9,6,7,8]$. 
Based on hybridization techniques we propose here a primal hybrid finite element method for linear elasticity combining the advantages of Discontinuous Galerkin methods with an element based data structure and reduced computational cost. As multiplier was chosen the displacement field itself and add stabilization and symmetrization terms to generate a stable and adjoint consistent formulation allowing greater flexibility in the choice of basis functions of approximation spaces for the displacement field and the Lagrange multiplier. The method is derived from a stabilized hybrid formulation consisting of a set of local problems defined at the element level coupled to a global problem on the multiplier only. we choose as multiplier the displacement field itself and add stabilization and symmetrization terms to generate a stable and adjoint consistent formulation allowing greater flexibility in the choice of basis functions of approximation spaces for the displacement field and the Lagrange multiplier.

The remainder of the paper is organized as follows. A review of notation required and the linear elasticity problem is presented in Section 2. The Interior Penalty Discontinuous Galerkin formulations is briefly presented in Section 3. The proposed Stabilized Hybridized primal formulation is introduced and discussed in Section 4. Numerical results on convergency studies are presented in Section 5. Concluding remarks are drawn in Section 6.

\section{PRELIMINARIES}

\subsection{Notation}

To introduce the stabilized hybrid formulation, we first present some definitions and notations. Let $\Omega \in \mathbb{R}^{d}, d \geq 1$, be a bounded domain with a Lipschitz boundary $\Gamma=\partial \Omega$, and $L^{2}(\Omega)$ the space of square integrable functions, equipped with the usual norm $\|\cdot\|_{0, \Omega}$. Let $H^{m}(\Omega)$ be the usual Sobolev space of all functions in $L^{2}(\Omega)$ whose weak derivatives up to the nonnegative integer order $m$ are also $L^{2}(\Omega)$-integrable [1]. The corresponding $H^{m}(\Omega)$ norms and semi-norms are denoted by $\|\cdot\|_{m, \Omega}=\|\cdot\|_{m}$ and $|\cdot|_{m, \Omega}=|\cdot|_{m}$, respectively.

For a given function space $V(\Omega)$, let $V(\Omega)^{d}$ and $V(\Omega)^{d \times d}$ be the spaces of all vector and tensor fields whose components belong to $V(\Omega)$. Without further specification, these spaces are furnished with the usual product norms (which, for simplicity, are denoted similarly as the norm in $V(\Omega)$ ). For vectors $\mathbf{v}, \mathbf{w} \in \mathbb{R}^{d}$, and matrices $\boldsymbol{\sigma}, \boldsymbol{\tau} \in \mathbb{R}^{d \times d}$, we use the standard notation

$$
(\operatorname{grad} \mathbf{v})_{i j}=\partial_{j} v_{i}, \quad(\operatorname{div} \boldsymbol{\sigma})_{i}=\sum_{j=1}^{d} \partial_{j} \sigma_{i j} \quad \text { and } \quad \boldsymbol{\sigma}: \boldsymbol{\tau}=\sum_{i, j=1}^{d} \sigma_{i j} \tau_{i j}
$$

Furthermore, let $\mathbf{v} \otimes \mathbf{w}$ be the matrix with components $(\mathbf{v} \otimes \mathbf{w})_{i j}=v_{i} w_{j}$. We will also use the identity

$$
\mathbf{v} \cdot \boldsymbol{\sigma} \mathbf{w}=\sum_{i, j=1}^{d} v_{i} \sigma_{i j} w_{j}=\boldsymbol{\sigma}:(\mathbf{v} \otimes \mathbf{w}) .
$$

For simplicity we restrict our finite element formulation to two dimension elasticity problems. Let

$$
\mathcal{T}_{h}=\{K\}:=\text { union of all elements } K
$$

be a regular finite element partition of the two-dimensional domain $\Omega$ and let

$$
\mathcal{E}_{h}=\left\{e: e \text { is an edge of } K \text { for all } K \in \mathcal{T}_{h}\right\}
$$


denote the set of all edges of all elements $K$ of the mesh $\mathcal{T}_{h}$.

$$
\mathcal{E}_{h}^{0}=\left\{e \in \mathcal{E}_{h} e \text { is an interior edge }\right\}
$$

is the set of interior edges, and

$$
\mathcal{E}_{h}^{\partial}=\mathcal{E}_{h} \cap \Gamma,
$$

the set of edges of $\mathcal{E}_{h}$ on the boundary of $\Omega$. We assume that the domain $\Omega$ is polygonal and $\mathcal{T}_{h}$ is a regular partition of $\Omega$. Thus, there exists $c>0$ such that $h \leq c h_{e}$, where $h_{e}$ is the diameter of the edge $e \in \partial K$ and $h$, the mesh parameter, is the maximum element diameter. For each edge $e$ we associate a unit normal vector $\mathbf{n}_{e}$.

For a scalar-valued function $v \in L^{2}(\Omega)$ with $\left.v\right|_{K} \in H^{m}(K)$ for all $K \in \mathcal{T}_{h}$, let $\|v\|_{m, h}$ be the usual broken $H^{m}$-type norm of $v$ defined by

$$
\|v\|_{m, h}=\left(\sum_{K \in \mathcal{T}_{h}}\|v\|_{m, K}^{2}\right)^{1 / 2} .
$$

If $\mathbf{v}$ is vector-valued or tensor-valued function, the corresponding term $\|\mathbf{v}\|_{m, h}$ is defined in a similar manner. For a vector or a tensor $\mathbf{v}$, denote by $|\mathbf{v}|$ the quantity $(\mathbf{v} \cdot \mathbf{v})^{1 / 2}$ or $(\mathbf{v}: \mathbf{v})^{1 / 2}$.

Let $K^{+}$and $K^{-}$be two adjacent elements of $\mathcal{T}_{h}$, $\mathbf{x}$ be an arbitrary point of the set $e=\partial K^{+} \cap \partial K^{-}, \mathbf{n}^{+}$and $\mathbf{n}^{-}$be the corresponding outward unit normals at that point. For a scalar-valued function, $q$, a vector-valued function, $\mathbf{v}$, or a matrix-valued function, $\tau$, that are smooth inside each element $K^{ \pm}$, let us denote by $\left(q^{ \pm}, \mathbf{v}^{ \pm}, \boldsymbol{\tau}^{ \pm}\right)$the traces of $(q, \mathbf{v}, \boldsymbol{\tau})$ on $e$ taken from within the interior of $K^{ \pm}$, respectively. Then we define averages at $\mathbf{x} \in e$ as follows:

$$
\left\{\{q\}=\frac{1}{2}\left(q^{+}+q^{-}\right), \quad\{\{\mathbf{v}\}\}=\frac{1}{2}\left(\mathbf{v}^{+}+\mathbf{v}^{-}\right), \quad\left\{\{\boldsymbol{\tau}\}=\frac{1}{2}\left(\boldsymbol{\tau}^{+}+\boldsymbol{\tau}^{-}\right) \text {on } e \in \mathcal{E}_{h}^{0} .\right.\right.
$$

Similarly, the jumps at $\mathbf{x} \in e$ on $e \in \mathcal{E}_{h}^{0}$ are given by

$$
\begin{aligned}
\llbracket p \rrbracket & =p^{+} \mathbf{n}^{+}+p^{-} \mathbf{n}^{-}, \\
\llbracket \mathbf{v} \rrbracket & =\mathbf{v}^{+} \cdot \mathbf{n}^{+}+\mathbf{v}^{-} \cdot \mathbf{n}^{-} \\
\llbracket \boldsymbol{\tau} \rrbracket & =\boldsymbol{\tau}^{+} \mathbf{n}^{+}+\boldsymbol{\tau}^{-} \mathbf{n}^{-}
\end{aligned}
$$

If $\mathbf{x}$ is on an edge $e$ lying on the boundary $\partial \Omega$, i.e., $e \in \mathcal{E}_{h}^{\partial}$, the above average and jump operators are defined by

$$
\begin{array}{ccc}
\{\{p\}=p, \quad\{\{\mathbf{v}\}=\mathbf{v}, \quad & \{\boldsymbol{\tau}\}=\boldsymbol{\tau}, \\
\llbracket p \rrbracket=p \mathbf{n}, & \llbracket \mathbf{v} \rrbracket=\mathbf{v} \cdot \mathbf{n}, \quad & \llbracket \boldsymbol{\tau} \rrbracket=\boldsymbol{\tau} \mathbf{n} .
\end{array}
$$

where $\mathbf{n}$ is the unit outward normal vector on $\partial \Omega$. We define a matrix-valued jump $\llbracket \cdot \rrbracket$ of a vector $\mathbf{v}$ as in [5]

$$
\begin{gathered}
\llbracket \mathbf{v} \rrbracket=\frac{1}{2}\left(\mathbf{v}^{+} \otimes \mathbf{n}^{+}+\mathbf{n}^{+} \otimes \mathbf{v}^{+}+\mathbf{v}^{-} \otimes \mathbf{n}^{-}+\mathbf{n}^{-} \otimes \mathbf{v}^{-}\right), \text {if } \mathbf{x} \in e \in \mathcal{E}_{h}^{0}, \\
\llbracket \mathbf{v} \rrbracket=\frac{1}{2}(\mathbf{v} \otimes \mathbf{n}+\mathbf{n} \otimes \mathbf{v}), \text { if } \mathbf{x} \in e \in \mathcal{E}_{h}^{\partial} .
\end{gathered}
$$




\subsection{Model problem}

Let $\Omega$ in $\mathbb{R}^{d}, d=2$ denote an open bounded domain with piecewise Lipschitz boundary $\Gamma=\Gamma_{D} \cup \Gamma_{N}=\partial \Omega$ of an elastic body subjected to external force $\mathbf{f} \in L^{2}(\Omega)^{2}$. The kinematical model of linear elasticity in two dimensions consists in finding a displacement vector field $\mathbf{u}$ satisfying

$$
\begin{aligned}
-\operatorname{div} \boldsymbol{\sigma}(\mathbf{u}) & =\mathbf{f} & & \text { in } \Omega, \\
\boldsymbol{\sigma}(\mathbf{u}) & =\mathbb{D} \boldsymbol{\varepsilon}(\mathbf{u}) & & \text { in } \Omega, \\
\mathbf{u} & =\mathbf{g} & & \text { on } \Gamma_{D} \\
\boldsymbol{\sigma}(\mathbf{u}) \mathbf{n} & =\mathbf{h} & & \text { on } \Gamma_{N}
\end{aligned}
$$

where $\boldsymbol{\sigma}(\mathbf{u})$ is the symmetric Cauchy stress tensor, $\boldsymbol{\varepsilon}(\mathbf{u})=\frac{1}{2}\left(\operatorname{grad} \mathbf{u}+\operatorname{grad} \mathbf{u}^{T}\right)$ is the linear strain tensor, $\mathbf{h}$ are the given boundary loads; $\mathbf{g}$ is a given boundary displacement and $\mathbf{n}$ is the exterior unit normal vector to $\Gamma[15,12]$. For linear, homogeneous and isotropic material $\boldsymbol{\sigma}(\mathbf{u})$ given by

$$
\boldsymbol{\sigma}(\mathbf{u})=\mathbb{D} \varepsilon(\mathbf{u})=2 \mu \varepsilon(\mathbf{u})+\lambda(\operatorname{tr} \varepsilon(\mathbf{u})) \mathbb{I},
$$

where $\operatorname{tr} \varepsilon(\mathbf{u})=\operatorname{div} \mathbf{u}, \mathbb{I}$ is the identity tensor and $\lambda$ and $\mu$ are called the Lamé parameters. In terms of the modulus of elasticity, $\mathrm{E}$, and Poisson's ratio, $\nu$, we have, in the case of plane strain, that

$$
\lambda=\frac{E \nu}{(1+\nu)(1-2 \nu)} \quad \text { and } \quad \mu=\frac{E}{2(1+\nu)} .
$$

In our model problem we will only consider homogeneous Dirichlet condition on $\Gamma_{D}=\Gamma$.

\section{PRIMAL DISCONTINUOUS GALERKIN FORMULATION}

In this section we recall interior penalty Discontinuous Galerkin formulations for our model problem $[19,5,13,17]$. Following Wihler [19], on the partition $\mathcal{T}_{h}$ we define the space

$$
\mathbf{V}=\left\{\mathbf{v} \in\left(L^{2}(\Omega)\right)^{2} ;\left.\mathbf{v}_{i}\right|_{K} \in H^{1}(K), \forall K \in \mathcal{T}_{h}, i=1,2\right\}
$$

The Symmetric Interior Penalty Discontinuous Galerkin (SIP-DG) method for our model problem is formulated as follows:

Find $\mathbf{u} \in \mathbf{V}$ such that,

$$
\begin{aligned}
\sum_{K \in \mathcal{T}_{h}} \int_{K} \mathbb{D} \boldsymbol{\varepsilon}(\mathbf{u}): \boldsymbol{\varepsilon}(\mathbf{v}) d x & -\sum_{K \in \mathcal{T}_{h}} \int_{\partial K} \mathbb{D} \boldsymbol{\varepsilon}(\mathbf{u}) \mathbf{n} \cdot \mathbf{v} d s-\sum_{K \in \mathcal{T}_{h}} \int_{\partial K} \mathbb{D} \boldsymbol{\varepsilon}(\mathbf{v}) \mathbf{n} \cdot\left(\mathbf{u}-\mathbf{u}^{\prime}\right) d s \\
& +\sum_{K \in \mathcal{T}_{h}} \int_{\partial K} \frac{\beta}{h}\left(\mathbf{u}-\mathbf{u}^{\prime}\right) \cdot \mathbf{v} d s=\sum_{K \in \mathcal{T}_{h}} \int_{K} \mathbf{f} \cdot \mathbf{v} d x, \quad \forall \mathbf{v} \in \mathbf{V},(4)
\end{aligned}
$$

with $\mathbf{u}^{\prime}=\left.\mathbf{u}\right|_{K^{\prime}}$ for all $K^{\prime} \in \mathcal{N}(K)$, the set of all neighbors of $K$, and $\mathbf{u}^{\prime}=\mathbf{u}=\mathbf{0}$ on $\Gamma$. The residual term has been consistently added according to the following Symmetric Interior Penalty Discontinuous Galerkin (SIP-DG) formulation. Discontinuous Galerkin methods are normally formulated and analyzed using well known average and jump operators defined on interior edges as in Section 2.1. Therefore, using the identity that has been demonstrated by Chen et al [5] (see Lemma 2.1)

$$
\sum_{K \in \mathcal{T}_{h}} \int_{\partial K}(\boldsymbol{\tau} \mathbf{n}) \cdot \mathbf{w} d s=\int_{\mathcal{E}_{h}^{0}} \llbracket \boldsymbol{\tau} \rrbracket \cdot\{\{\mathbf{w}\}\}+\{\{\boldsymbol{\tau}\}: \llbracket \mathbf{w} \rrbracket d s
$$


this class of Discontinuous Galerkin method can be formulated as [19]:

Find $\mathbf{u} \in \mathbf{V}$ such that

$$
a_{D G}(\mathbf{u}, \mathbf{v})=f(\mathbf{v}) \quad \forall \mathbf{v} \in \mathbf{V}
$$

with

$$
\begin{aligned}
& a_{D G}(\mathbf{u}, \mathbf{v})=\sum_{K \in \mathcal{T}_{h}} \int_{K} \mathbb{D} \varepsilon(\mathbf{u}): \varepsilon(\mathbf{v}) d x-\sum_{e \in \mathcal{E}_{h}} \int_{e}(\{\{\mathbb{D} \varepsilon(\mathbf{u})\}: \llbracket \mathbf{v} \rrbracket+\llbracket \mathbf{u} \rrbracket:\{\{\mathbb{D} \varepsilon(\mathbf{v})\}\}) d s \\
& +\sum_{e \in \mathcal{E}_{h}^{0}} \int_{e} \frac{\beta}{h} \llbracket \mathbf{u} \rrbracket: \llbracket \mathbf{v} \rrbracket d s+\sum_{e \in \mathcal{E}_{h}^{\partial}} \int_{e} \frac{\beta}{h} \llbracket \mathbf{u} \rrbracket: \llbracket \mathbf{v} \rrbracket d s, \\
& f(\mathbf{v})=\sum_{K \in \mathcal{T}_{h}} \int_{K} \mathbf{f} \cdot \mathbf{v} d x .
\end{aligned}
$$

The numerical analysis of this discontinuous Galerkin methods is provided in reference [13] following the unified framework for the analysis of DG methods introduced in [3] for closely related diffusion problems.

\section{STABILIZED HYBRID FORMULATION}

\subsection{Stabilized Hybrid Discontinuous Finite Element Method}

We now present a Stabilized Hybrid Discontinuous formulation for the linear elasticity problem in its primal form with the multiplier $\boldsymbol{\lambda}$ defined as the trace of $\mathbf{u}: \boldsymbol{\lambda}=\left.\mathbf{u}\right|_{e}$ on each edge $e \in \mathcal{E}_{h}$. This presentation will be restricted to the finite dimension spaces:

$$
\begin{gathered}
\mathbf{V}_{h}=\left\{\mathbf{v} \in\left(L^{2}(\Omega)\right)^{2}:\left.\mathbf{v}\right|_{K} \in\left[S_{k}(K)\right]^{2} \quad \forall K \in \mathcal{T}_{h}\right\} \\
\mathbf{M}_{h}=\left\{\boldsymbol{\lambda} \in\left(L^{2}\left(\mathcal{E}_{h}\right)\right)^{2}:\left.\boldsymbol{\lambda}\right|_{e}=\left[P_{l}(e)\right]^{2}, \forall e \in \mathcal{E}_{h}^{0},\left.\boldsymbol{\lambda}\right|_{e}=0, \forall e \in \mathcal{E}_{h}^{\partial}\right\},
\end{gathered}
$$

where $S_{k}(K)=P_{k}(K)$ (the space of polynomial functions of degree at most $k$ in both variables) or $S_{k}(K)=Q_{k}(K)$ (the space of polynomial functions of degree at most $k$ in any variable), and $P_{l}(e)$ is the space of of polynomials of degree at most $l$ on each edge $e$. These finite dimension spaces are usually associated with triangular or quadrilateral elements, however the proposed formulation can be naturally applied to any finite element partition consisting of general polygons. The Stabilized Hybrid Discontinuous Galerkin method is formulated as:

Find the pair $\left(\mathbf{u}_{h}, \boldsymbol{\lambda}_{h}\right) \in \mathbf{V}_{h} \times \mathbf{M}_{h}$ such that for all $\left(\mathbf{v}_{h}, \boldsymbol{\mu}_{h}\right) \in \mathbf{V}_{h} \times \mathbf{M}_{h}$

$$
\begin{array}{r}
\sum_{K \in \mathcal{T}_{h}} \int_{K} \mathbb{D} \varepsilon\left(\mathbf{u}_{h}\right): \varepsilon\left(\mathbf{v}_{h}\right) d x-\sum_{K \in \mathcal{T}_{h}} \int_{\partial K}\left[\left(\mathbb{D} \boldsymbol{\varepsilon}\left(\mathbf{u}_{h}\right) \mathbf{n}\right) \cdot \mathbf{v}_{h}+\left(\mathbb{D} \boldsymbol{\varepsilon}\left(\mathbf{v}_{h}\right) \mathbf{n}\right) \cdot\left(\mathbf{u}_{h}-\boldsymbol{\lambda}_{h}\right)\right] d s \\
+\sum_{K \in \mathcal{T}_{h}} \int_{\partial K} \frac{\beta}{h}\left(\mathbf{u}_{h}-\boldsymbol{\lambda}_{h}\right) \cdot \mathbf{v}_{h} d s=\sum_{K \in \mathcal{T}_{h}} \int_{K} \mathbf{f} \cdot \mathbf{v}_{h} d x, \quad \forall \mathbf{v}_{h} \in \mathbf{V}_{h},
\end{array}
$$

$$
\sum_{K \in \mathcal{T}_{h}} \int_{\partial K}\left(\mathbb{D} \boldsymbol{\varepsilon}\left(\mathbf{u}_{h}\right) \mathbf{n}\right) \cdot \boldsymbol{\mu}_{h} d s+\sum_{K \in \mathcal{T}_{h}} \int_{\partial K} \frac{\beta}{h}\left(\boldsymbol{\lambda}_{h}-\mathbf{u}_{h}\right) \cdot \boldsymbol{\mu}_{h} d s=0, \quad \forall \boldsymbol{\mu}_{h} \in \mathbf{M}_{h}
$$


In the above formulation the boundary condition $\mathbf{u}=\mathbf{0}$ on $\Gamma$ is weakly imposed using the same Nitsche's approach adopted in the associate DG method. For this reason the unknown $\boldsymbol{\lambda}_{h}$ is restricted to $\mathbf{M}_{h}$ and on each $e \in \mathcal{E}_{h}^{\partial}$ we set $\boldsymbol{\lambda}_{h}=\mathbf{0}$. Also, a symmetrization term to generate a stable and adjoint consistent formulation allowing greater flexibility in the choice of basis functions of the approximation spaces for the displacement field and the Lagrange multiplier was added. Here, $\beta$ is a penalty parameter introduced to stabilize the displacement field $\mathbf{u}_{h}$ and the multiplier $\boldsymbol{\lambda}_{h}$ and has dimension equal to the Young module. In the next sections we show that the proposed Stabilized Hybrid formulation preserves basically the same properties of the corresponding Symmetric Interior Penalty Discontinuous Galerkin method.

\subsection{Consistency}

The pair $(\mathbf{u}, \boldsymbol{\lambda})$, with $\mathbf{u}$ solution of the model problem (1) and $\boldsymbol{\lambda}=\left.\mathbf{u}\right|_{e}$ on each edge $e \in \mathcal{E}_{h}$, satisfies

$$
\begin{aligned}
& \sum_{K \in \mathcal{T}_{h}} \int_{K} \mathbb{D} \varepsilon(\mathbf{u}): \boldsymbol{\varepsilon}\left(\mathbf{v}_{h}\right) d x-\sum_{K \in \mathcal{T}_{h}} \int_{\partial K}(\mathbb{D} \boldsymbol{\varepsilon}(\mathbf{u}) \mathbf{n}) \cdot\left(\mathbf{v}_{h}-\boldsymbol{\mu}_{h}\right) d s \\
& -\sum_{K \in \mathcal{T}_{h}} \int_{\partial K}\left(\mathbb{D} \boldsymbol{\varepsilon}\left(v_{h}\right) \mathbf{n}\right) \cdot(\mathbf{u}-\boldsymbol{\lambda}) d s+\sum_{K \in \mathcal{T}_{h}} \int_{\partial K} \frac{\beta}{h}(\mathbf{u}-\boldsymbol{\lambda}) \cdot\left(\mathbf{v}_{h}-\boldsymbol{\mu}_{h}\right) d s=\sum_{K \in \mathcal{T}_{h}} \int_{K} \mathbf{f} \cdot \mathbf{v}_{h} d s
\end{aligned}
$$

for all $\left\{\mathbf{v}_{h}, \boldsymbol{\mu}_{h}\right\} \in \mathbf{V}_{h} \times \mathbf{M}_{h}$.

Given that $\mathbf{u} \in\left[H^{2}(\Omega)\right]^{2}$, then $\boldsymbol{\lambda}=\left.\{\mathbf{u}\}\right|_{e}=\left.\mathbf{u}\right|_{e}$ and $\left.\llbracket \mathbb{D} \boldsymbol{\varepsilon}(\mathbf{u}) \rrbracket\right|_{e}=\mathbf{0}, \forall e \in \mathcal{E}_{h}^{0}$, equation (13) reduces to

$$
\sum_{K \in \mathcal{T}_{h}} \int_{K} \mathbb{D} \boldsymbol{\varepsilon}(\mathbf{u}): \varepsilon\left(\mathbf{v}_{h}\right) d x-\sum_{K \in \mathcal{T}_{h}} \int_{\partial K}(\mathbb{D} \boldsymbol{\varepsilon}(\mathbf{u}) \mathbf{n}) \cdot \mathbf{v}_{h} d s=\sum_{K \in \mathcal{T}_{h}} \int_{K} \mathbf{f} \cdot \mathbf{v}_{h} d s \quad \forall \mathbf{v}_{h} \in \mathbf{V}_{h}
$$

which integrated by parts leads to

$$
\sum_{K \in \mathcal{T}_{h}} \int_{K}[-\operatorname{div}(\mathbb{D} \boldsymbol{\varepsilon}(\mathbf{u}))-\mathbf{f}] \cdot \mathbf{v}_{h} d x=0 \quad \forall v_{h} \in \mathbf{V}_{h}
$$

proving consistency.

\subsection{A stabilized DG formulation}

To compare the stabilized hybrid formulation with the Interior Penalty Discontinuous Galerkin method analyzed by Hansbo et al [13] we consider the option of eliminating the multiplier at the level of an edge $e$ on each element $K \in T c$ as follows. For $l \geq k$, we can solve equation (12) exactly, obtaining

$$
\boldsymbol{\lambda}_{h}=\left\{\mathbf{u}_{h}\right\}-\frac{h}{2 \beta} \llbracket \mathbb{D} \boldsymbol{\varepsilon}\left(\mathbf{u}_{h}\right) \rrbracket \text { on each interior edge } e \in \mathcal{E}_{h}^{0} .
$$

Replacing (16) in (11) and using identities

$$
\begin{aligned}
& \mathbf{u}_{1}=\frac{1}{2}\left(\mathbf{u}_{1}+\mathbf{u}_{2}\right)+\frac{1}{2}\left(\mathbf{u}_{1}-\mathbf{u}_{2}\right), \\
& \mathbf{u}_{2}=\frac{1}{2}\left(\mathbf{u}_{1}+\mathbf{u}_{2}\right)-\frac{1}{2}\left(\mathbf{u}_{1}-\mathbf{u}_{2}\right),
\end{aligned}
$$


and (5), we obtain the following stabilized DG formulation:

Find $\mathbf{u}_{h} \in \mathbf{V}_{h}$ such that

$$
a_{S H}\left(\mathbf{u}_{h}, \mathbf{v}_{h}\right)=f\left(\mathbf{v}_{h}\right) \quad \forall \mathbf{v}_{h} \in \mathbf{V}_{h}
$$

with

$$
\begin{aligned}
a_{S H}\left(\mathbf{u}_{h}, \mathbf{v}_{h}\right) & =\sum_{K \in \mathcal{T}_{h}} \int_{K} \mathbb{D} \varepsilon\left(\mathbf{u}_{h}\right): \varepsilon\left(\mathbf{v}_{h}\right) d x-\sum_{e \in \mathcal{E}_{h}} \int_{e}\left\{\mathbb{D} \boldsymbol{\varepsilon}\left(\mathbf{u}_{h}\right)\right\}: \llbracket \mathbf{v}_{h} \rrbracket d s \\
& -\sum_{e \in \mathcal{E}_{h}} \int_{e}\left\{\mathbb{D} \boldsymbol{\varepsilon}\left(\mathbf{v}_{h}\right)\right\}: \llbracket \mathbf{u}_{h} \rrbracket d s-\frac{h}{2 \beta} \sum_{e \in \mathcal{E}_{h}^{0}} \int_{e} \llbracket \mathbb{D} \varepsilon\left(\mathbf{u}_{h}\right) \rrbracket \cdot \llbracket \mathbb{D} \boldsymbol{\varepsilon}\left(\mathbf{v}_{h}\right) \rrbracket d s \\
& +\frac{\beta}{2 h} \sum_{e \in \mathcal{E}_{h}^{0}} \int_{e} \llbracket \mathbf{u} \rrbracket: \llbracket \mathbf{v} \rrbracket d s+\frac{\beta}{h} \sum_{e \in \mathcal{E}_{h}^{\partial}} \int_{e} \llbracket \mathbf{u} \rrbracket: \llbracket \mathbf{v} \rrbracket d s .
\end{aligned}
$$

Comparing the bilinear for $a_{S H}(\cdot, \cdot)$ with the bilinear form $a_{D G}(\cdot, \cdot)$ corresponding to the DG formulation (6) we observe that the Stabilized Hybrid Discontinuous formulation is not an exact hybridization of this DG formulation. The only difference between these bilinear forms is the presence of the term

$$
-\frac{h}{2 \beta} \sum_{e \in \mathcal{E}_{h}^{0}} \int_{e} \llbracket \mathbb{D} \varepsilon\left(\mathbf{u}_{h}\right) \rrbracket \cdot \llbracket \mathbb{D} \varepsilon\left(\mathbf{v}_{h}\right) \rrbracket d s
$$

in the bilinear for $a_{S H}(\cdot, \cdot)$. Clearly, this term (20) does not affect the consistency of the the DG method (18) derived from the hybrid formulation. We also observe that, for sufficiently large $\beta$, stability and continuity of $a_{S H}(\cdot, \cdot)$ is proved using the same arguments adopted for proving these properties for the bilinear form $a_{D G}(\cdot, \cdot)$. Consequently, the error estimates derived for the DG method, as in references $[2,16]$, are applicable to the stabilized DG formulation (18). From the computational point of view eliminating the multiplier $\boldsymbol{\lambda}_{h}$ in favor of the discontinuous displacement field $\mathbf{u}_{h}$ is not a good choice. However, this decoupling strategy is very interesting concerning numerical analysis and error estimates of the proposed stabilized hybrid formulation. In the next section we present a different strategy to decouple the hybrid system consisting in eliminate the discontinuous displacement field $\mathbf{u}_{h}$ at the element level and assembling a global system involving only the multiplier.

\subsection{Hybridization}

Given that the multiplier of the proposed formulation is identified with the trace of the primal variable $\mathbf{u}$ on the element edges, we observe that, for $\beta$ sufficiently large, we can always eliminate the degrees-of-freedom of the primal variable $\mathbf{u}_{h}$ at the element level in favor of the degrees-of-freedom of the multiplier leading to a global system in the multiplier only. Computationally this is the best choice for leading to a global system with a much smaller dimension compared to the DG global system in $\mathbf{u}_{h}$.

Considering that $\mathbf{v}_{h}$, belonging to the broken function space $\mathbf{V}_{h}$, is defined independently on each element $K \in \mathcal{T}_{h}$, we observe that equation (11) can be split into a set of local problems defined on each element $K$ coupled to the global problem (11) defined on $\mathcal{E}_{h}$, as follow: 
Find $\left.\mathbf{u}_{h}\right|_{K} \in \mathbf{V}_{h}(K)=\left.\mathbf{V}_{h}\right|_{K}$ and $\lambda_{h} \in \mathbf{M}_{h}$, such that

$$
\begin{aligned}
& \int_{K} \mathbb{D} \varepsilon\left(\mathbf{u}_{h}\right): \varepsilon\left(\mathbf{v}_{h}\right) d x-\int_{\partial K}\left(\mathbb{D} \varepsilon\left(\mathbf{u}_{h}\right) \mathbf{n}\right) \cdot \mathbf{v}_{h} d s-\int_{\partial K}\left(\mathbb{D} \varepsilon\left(\mathbf{v}_{h}\right) \mathbf{n}\right) \cdot\left(\mathbf{u}_{h}-\boldsymbol{\lambda}_{h}\right) d s \\
& +\int_{\partial K} \frac{\beta}{h}\left(\mathbf{u}_{h}-\boldsymbol{\lambda}_{h}\right) \cdot \mathbf{v}_{h} d s=\int_{K} \mathbf{f} \cdot \mathbf{v}_{h} d x,\left.\quad \forall \mathbf{v}_{h}\right|_{K} \in \mathbf{V}_{h}(K), \\
& \sum_{K \in \mathcal{T}_{h}} \int_{\partial K}\left(\mathbb{D} \boldsymbol{\varepsilon}\left(\mathbf{u}_{h}\right) \mathbf{n}\right) \cdot \boldsymbol{\mu}_{h} d s+\sum_{K \in \mathcal{T}_{h}} \int_{\partial K} \frac{\beta}{h}\left(\boldsymbol{\lambda}_{h}-\mathbf{u}_{h}\right) \cdot \boldsymbol{\mu}_{h} d s=0, \quad \forall \boldsymbol{\mu}_{h} \in \mathbf{M}_{h}
\end{aligned}
$$

Defining the local bilinear forms:

$$
\begin{aligned}
a_{K}\left(\mathbf{u}_{h}, \mathbf{v}_{h}\right) & =\int_{K} \mathbb{D} \varepsilon\left(\mathbf{u}_{h}\right): \varepsilon\left(\mathbf{v}_{h}\right) d x \\
& -\int_{\partial K}\left(\mathbb{D} \varepsilon\left(\mathbf{u}_{h}\right) \mathbf{n}\right) \cdot \mathbf{v}_{h} d s-\int_{\partial K}\left(\mathbb{D} \varepsilon\left(\mathbf{v}_{h}\right) \mathbf{n}\right) \cdot \mathbf{u}_{h} d s+\frac{\beta}{h} \int_{\partial K} \mathbf{u}_{h} \cdot \mathbf{v}_{h} d s ; \\
b_{K}\left(\lambda_{h}, \mathbf{v}_{h}\right) & =-\int_{\partial K}\left(\mathbb{D} \varepsilon\left(\mathbf{v}_{h}\right) \mathbf{n}\right) \cdot \boldsymbol{\lambda}_{h} d s-\frac{\beta}{h} \int_{\partial K} \boldsymbol{\lambda}_{h} \cdot \mathbf{v}_{h} d s \\
c_{K}\left(\boldsymbol{\lambda}_{h}, \boldsymbol{\mu}_{h}\right) & =\frac{\beta}{h} \int_{\partial K} \boldsymbol{\lambda}_{h} \cdot \boldsymbol{\mu}_{h} d s
\end{aligned}
$$

and the linear functional

$$
f_{K}\left(\mathbf{v}_{h}\right)=\int_{K} \mathbf{f} \cdot \mathbf{v}_{h} d x
$$

the system (21)-(22) can be presented as:

Find $\left.\mathbf{u}_{h}\right|_{K} \in \mathbf{V}_{k}(K)$, for each $K \in \mathcal{T}_{h}$, and $\boldsymbol{\lambda}_{h} \in M_{h}$ such that

$$
\begin{aligned}
& a_{K}\left(\mathbf{u}_{h}, \mathbf{v}_{h}\right)+b_{K}\left(\boldsymbol{\lambda}_{h}, \mathbf{v}_{h}\right)=f_{K}\left(\mathbf{v}_{h}\right), \quad \forall \mathbf{v}_{h} \in \mathbf{V}_{k}(K), \\
& \sum_{K \in \mathcal{T}_{h}} b_{K}^{T}\left(\mathbf{u}_{h}, \boldsymbol{\mu}_{h}\right)+\sum_{K \in \mathcal{T}_{h}} c_{K}\left(\boldsymbol{\lambda}_{h}, \boldsymbol{\mu}_{h}\right)=\mathbf{0}, \quad \forall \boldsymbol{\mu}_{h} \in \mathbf{M}_{h},
\end{aligned}
$$

or in matrix form:

$$
\begin{gathered}
\mathbf{A}_{K} \mathbf{U}+\mathbf{B}_{K} \boldsymbol{\Lambda}=\mathbf{F}_{K}, \quad \forall K \in \mathcal{T}_{h} \\
\sum_{K \in \mathcal{T}_{h}} \mathbf{B}_{K}^{T} \mathbf{U}+\sum_{K \in \mathcal{T}_{h}} \mathbf{C}_{K} \boldsymbol{\Lambda}=\mathbf{0} .
\end{gathered}
$$

Inverting the local matrix $\mathbf{A}_{K}$ we have

$$
\mathbf{U}=\mathbf{A}_{K}^{-1}\left(\mathbf{F}_{K}-\mathbf{B}_{K} \boldsymbol{\Lambda}\right), \quad \forall K \in \mathcal{T}_{h}
$$

Replacing (31) in (30), we obtain the global system in the multiplier only:

$$
\sum_{K \in \mathcal{T}_{h}}\left(\mathbf{C}_{K}-\mathbf{B}_{K}^{T} \mathbf{A}_{K}^{-1} \mathbf{B}_{K}\right) \boldsymbol{\Lambda}=\sum_{K \in \mathcal{T}_{h}}-\mathbf{B}_{K}^{T} \mathbf{A}_{K}^{-1} \mathbf{F}_{K}
$$

After solving the global (32), the vector $\mathbf{U}$ is obtained from (31) with $\Lambda$ given by (32). 


\section{NUMERICAL RESULTS}

In this section the proposed formulation is validated on some numerical experiments. The stabilized hybrid system of Eq. (21-22) is solved using the strategy presented in (31-32). The performance of the method is tested by presenting some results of convergence studies for plane-strain problem defined on square domain $\Omega=(0,1) \times(0,1)$ with homogeneous boundary conditions, considering elasticity modulus $E=1$, Poisson ratio $\nu=0.3$ and forcing term:

$$
\begin{aligned}
& f_{1}(x, y)=\mu \cos (\pi x-\pi y)-2 \mu \cos (\pi x+\pi y)-\lambda \cos (\pi x+\pi y) \\
& f_{2}(x, y)=\mu \cos (\pi x-\pi y)-2 \mu \cos (\pi x+\pi y)-\lambda \cos (\pi x+\pi y)
\end{aligned}
$$

such that the exact solution is given by

$$
\begin{aligned}
& u_{1}(x, y)=\frac{1}{\pi^{2}} \sin (\pi x) \sin (\pi y) \\
& u_{2}(x, y)=\frac{1}{\pi^{2}} \sin (\pi x) \sin (\pi y) .
\end{aligned}
$$

In this study we will compare the stabilized hybrid approximation $\mathbf{u}_{h}$ with the continuous interpolant $\mathbf{u}_{I}$ and with the following Local Projections of the exact solution:

For given $\boldsymbol{\lambda}=\left.\mathbf{u}\right|_{\partial K}$, the trace of the exact solution on the edge of the element $K$, find $\mathbf{u}_{L P} \in \mathbf{V}_{h}(K)$ such that

$$
a_{K}\left(\mathbf{u}_{L P}, \mathbf{v}_{h}\right)=f_{K}\left(\mathbf{v}_{h}\right)-b_{K}\left(\boldsymbol{\lambda}, \mathbf{v}_{h}\right), \quad \forall \mathbf{v}_{h} \in \mathbf{V}_{k}(K), \quad \text { for each element } K \in \mathcal{T}_{h}
$$

Figure 1 presents h-convergence comparisons of the stabilized hybrid approximation $\mathbf{u}_{h}$ with the Local Projection $\mathbf{u}_{L P}$ and the interpolant $\mathbf{u}_{I}$ for $k=l=1$ and $\beta=8$. In this study we adopted a sequence of $2 \times 2,4 \times 4,8 \times 8$ and $16 \times 16$ uniform finite element meshes. Optimal rates of convergence are observed for all approximations in both $H^{1}(\Omega)$ seminorm $(\mathcal{O}(h))$ and $L^{2}(\Omega)$ norm $\left(\mathcal{O}\left(h^{2}\right)\right.$ ), with identical accuracy in $H^{1}(\Omega)$ seminorm for all approximations. In $L^{2}(\Omega)$ the stabilized hybrid approximation $\mathbf{u}_{h}$ coincides with the Local Projection and is more accurate than the corresponding interpolant.

In Figure $2 \mathrm{~h}$-convergence results are presented for $k=l=2$ and $\beta=16$. In this cases, optimal rates of convergence are also observed for all approximations in both $H^{1}(\Omega)$ seminorm $\left(\mathcal{O}\left(h^{2}\right)\right)$ and $L^{2}(\Omega)$ norm $\left(\mathcal{O}\left(h^{3}\right)\right)$. Again, we observe a great coincidence between the hybrid approximation and the Local Projection with improved accuracy in $L^{2}(\Omega)$ compared to the interpolant.

Figure 3 presents h-convergence studies for the multiplier $\lambda_{h}$ compared to the corresponding interpolant $\boldsymbol{\lambda}_{I}$ for $k=l=1$ (left) and $k=l=2$ (right). Optimal rates of convergence of $\mathcal{O}\left(h^{k+0.5}\right)$ in $L^{2}\left(\mathcal{E}_{h}\right)$ norm are again observed in both cases.

Figure 4 presents results of p-convergence study for the stabilized hybrid approximation $\mathbf{u}_{h}$ compared to the Local projection $\mathbf{u}_{L P}$ and the interpolant $\mathbf{u}_{I}$. In this study we adopted a uniform mesh with four quadrilateral elements and considered $k=l=1,2,3,4,5$, with $\beta=8,16,26,42,170$, respectively. In the $H^{1}(\Omega)$ seminorm (left) the three solution practically coincide. In $L^{2}(\Omega)$ norm we observe that the stabilized hybrid approximations and the Local Projection are more accurate than the interpolant for $k=l=1,2,3$. 

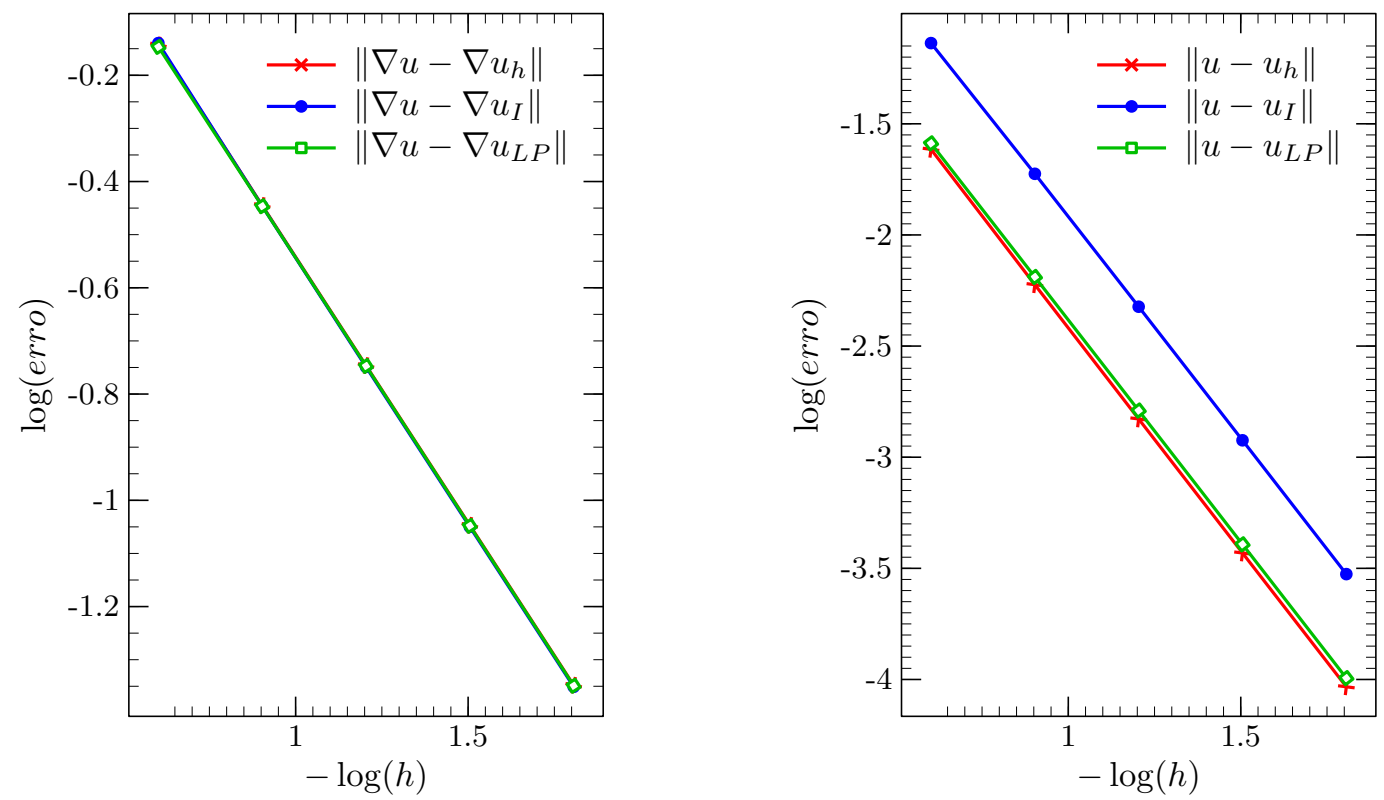

Figure 1. h-Convergence of Stabilized Hybrid Discontinuous Galerkin approximation $\left(\mathbf{u}_{h}\right)$ compared Local Projection $\left(\mathbf{u}_{L P}\right)$ and the Interpolant $\left(\mathbf{u}_{I}\right)$ for Q1Q1-2 in $H^{1}(\Omega)$ seminorm (left) and $L^{2}(\Omega)$ norm (right).
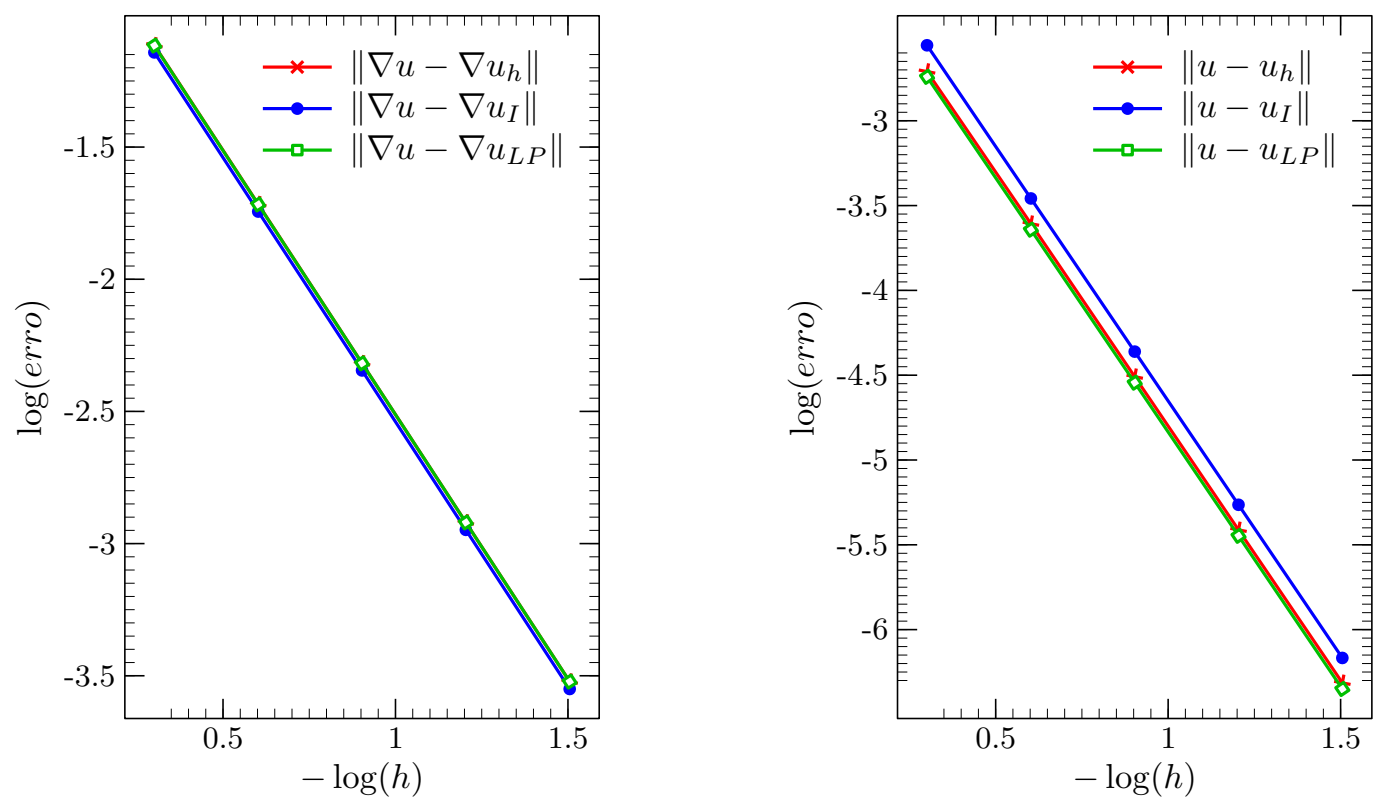

Figure 2. h-Convergence of Stabilized Hybrid Discontinuous Galerkin approximation $\left(\mathbf{u}_{h}\right)$ compared Local Projection $\left(\mathbf{u}_{L P}\right)$ and the Interpolant $\left(\mathbf{u}_{I}\right)$ for Q2Q2-3 in $H^{1}(\Omega)$ seminorm (left) and $L^{2}(\Omega)$ norm (right). 

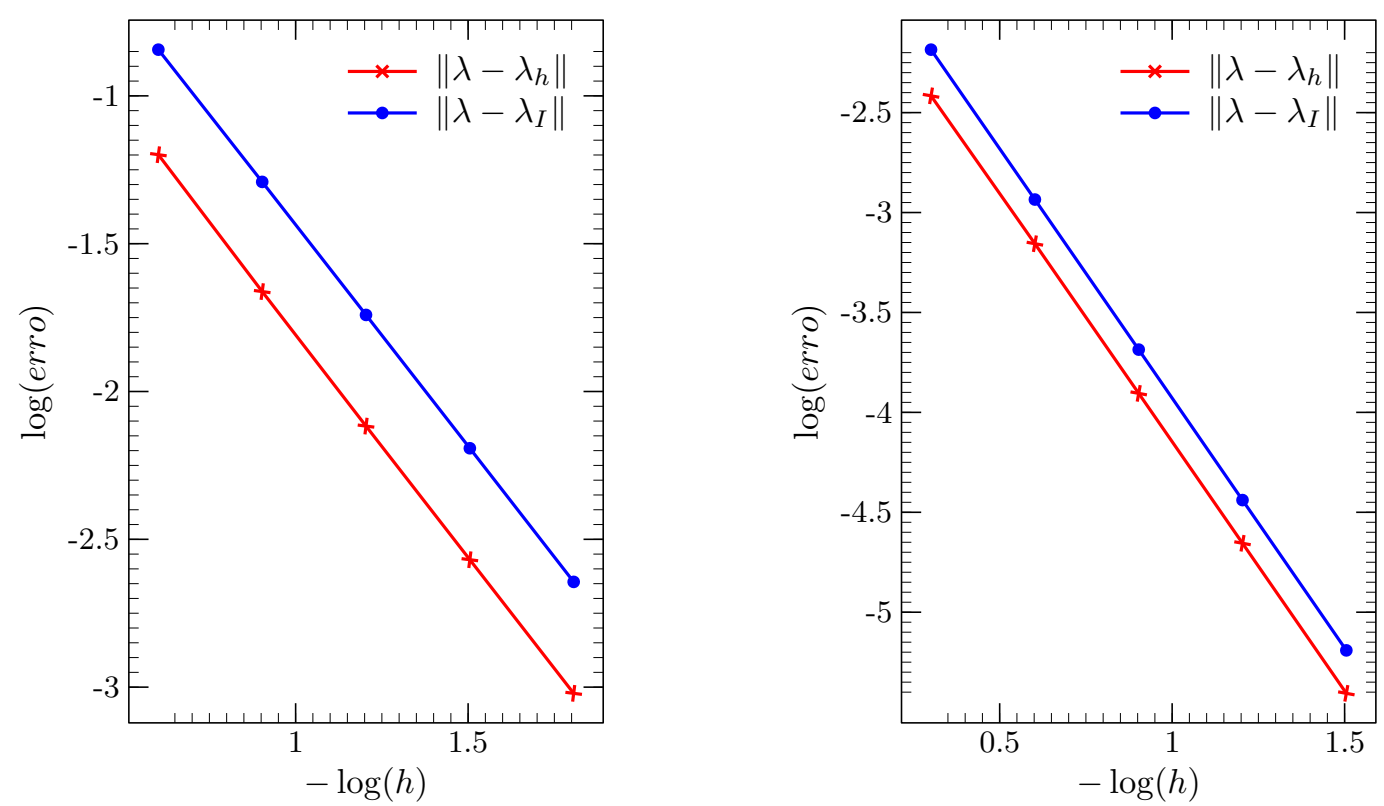

Figure 3. h-Convergence of Stabilized Hybrid Discontinuous Galerkin approximation $\left(\boldsymbol{\lambda}_{h}\right)$ compared the Interpolant $\left(\boldsymbol{\lambda}_{I}\right)$ in $L^{2}(\Omega)$ norm for Q1Q1-2 (left) and Q2Q2-3 (right).
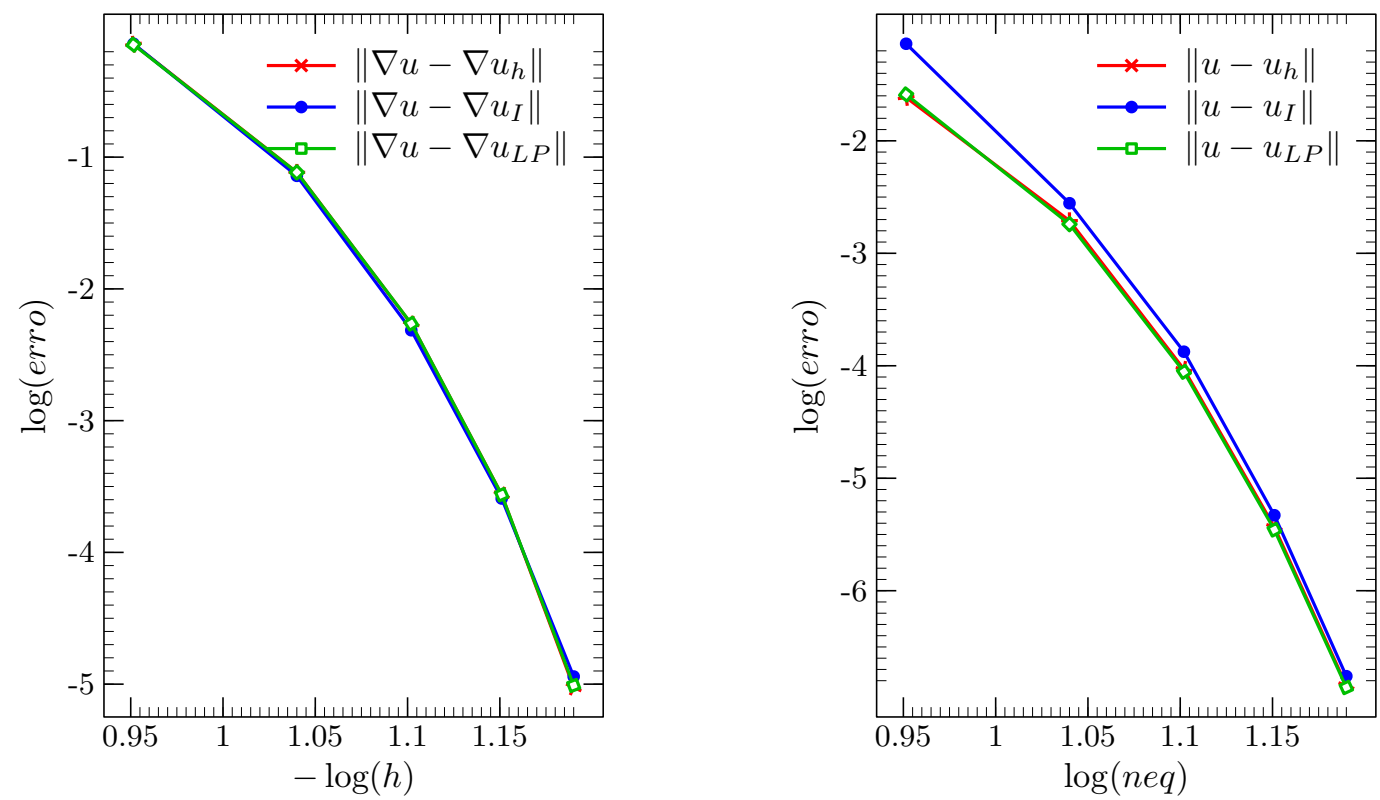

Figure 4. p-Convergence of Stabilized Hybrid Discontinuous Galerkin approximation $\left(u_{h}\right)$ compared the Local Projection $\left(u_{L P}\right)$ and the Interpolant in $H^{1}(\Omega)$ seminorm (left) and $L^{2}(\Omega)$ norm (right). 


\section{CONCLUDING REMARKS}

A Stabilized Hybrid Discontinuous Galerkin finite element formulation for linear elasticity problems is proposed. The method is developed from a primal hybrid formulation with the Lagrange multiplier identified with the trace of the displacement field on the edges of the elements leading to a set of local problems defined at the element level and a global problem in the multiplier only. We show that the proposed formulation preserves the main properties of the corresponding Interior Penalty Discontinuous Galerkin method but with reduced computational cost. The proposed method is easily implemented using the same data structure of continuous Galerkin finite element methods, allowing different degrees of interpolation polynomials for the primal variable and for the multiplier, giving rise to more flexibility and improved accuracy. Numerical results on a convergence study show optimal rates of convergence for the primal variable $\mathbf{u}_{h}$ and for the Lagrange multiplier $\boldsymbol{\lambda}_{h}$.

\section{Acknowledgements}

The authors thank the Brazilian Research Council (CNPq) and the Rio de Janeiro State Foundation (FAPERJ) for their financial support to the development of this work.

\section{REFERENCES}

[1] R. Adams. Sobolev Spaces. Academic Press, New York, 1975.

[2] D. N. Arnold. An interior penalty finite element method with discontinuous elements. SIAM J. Numer. Anal., 19(4):742-760, 1982.

[3] D. N. Arnold, F. Brezzi, B. Cockburn, and L. D. Marini. Unified analysis of discontinuous Galerkin methods for elliptic problems. SIAM J. Numer. Anal., 39(5):1749-1779, 2001/02.

[4] F. Brezzi and M. Fortin. Mixed and Hybrid Finite Element Methods. Springer-Verlag, 1991.

[5] Y. Chen, J. Huang, X. Huang, and Y. Xu. On the Local Discontinuous Galerkin method for linear elasticity. Mathematical Problems in Engineering, 2010:20 pages, 2010.

[6] B. Cockburn, B. Dong, and J. Guzmán. A superconvergent LDG-hybridizable Galerkin method for second-order elliptic problems. Math. Comp., 77(264):1887-1916, 2008.

[7] B. Cockburn, B. Dong, J. Guzmán, M. Restelli, and R. Sacco. A hybridizable discontinuous Galerkin method for steady-state convection-diffusion-reaction problems. SIAM J. Sci. Comput., 31(5):3827-3846, 2009.

[8] B. Cockburn, J. Gopalakrishnan, and R. Lazarov. Unified hybridization of discontinuous galerkin, mixed, and continuous galerkin methods for second order elliptic problems. 47(2):1319-1365, 2009.

[9] B. Cockburn, J. Gopalakrishnan, and F.-J. Sayas. A projection-based error analysis of HDG methods. Math. Comp., 79(271):1351-1367, 2010. 
[10] L. P. Franca, T. J. R. Hughes, A. F. D. Loula, and I. Miranda. A new family of stable elements for nearly incompressible elasticity based on a mixed Petrov-Galerkin finite element formulation. Numerische Mathematik, 53:123-141, 1988.

[11] L. P. Franca and R. Stenberg. Error analysis of some Galerkin least squares methods for the elasticity equations. SIAM J. Numer. Anal., 28(6):1680-1697, 1991.

[12] M. E. Gurtin. An Introductory to Continuum Mechanics. Academic Press, New York, NY, 1981.

[13] P. Hansbo and M. G. Larson. Discontinuous Galerkin methods for incompressible and nearly incompressible elasticity by Nitsche's method. Comput. Methods Appl. Mech. Engrg., 191:1895-1908, 2002.

[14] R. Kouhia and R. Stenberg. A linear nonconforming finite element method for nearly incompressible elasticity and stokes flow. Comput. Methods Appl. Mech. Engrg., 124(3):195-212, 1995.

[15] W. M. Lai, D. Rubin, and E. Krempl. Introductory to Continuum Mechanics. Butterworth Heinemann, 3rd Edition, Woburn, MA, 1999.

[16] B. Rivière. Discontinuous Galerkin Methods for Solving Elliptic and Parabolic Equations: theory and implementation. SIAM, 2008.

[17] B. Rivière and M. F. Wheeler. Optimal error estimates for discontiuous Galerkin methods applied to linear elasticity problems. Comput. Math. Appl, 46:141-163, 2000.

[18] M. Vogelius. An analysis of the p-version of the finite element method for nearly incompressible materials. Numer. Math., 41:39-53, 1983.

[19] T. P. Wihler. Locking-free adaptive discontinuous Galerkin FEM for linear elasticity problems. Mathematics of Computation, 75(255):1087-1102, 2006. 